\title{
Mechanical Properties of Polyurethane/Montmorillonite Nanocomposite Prepared by Melt Mixing
}

\author{
Byoung Chul Chun, ${ }^{1,2}$ Tae Keun Cho, ${ }^{1,2}$ Mi Hwa Chong, ${ }^{1,2}$ Yong-Chan Chung, ${ }^{2,3}$ Jihua Chen, ${ }^{4}$ \\ David Martin, ${ }^{4}$ Robert C. Cieslinski ${ }^{5}$ \\ ${ }^{1}$ Department of Polymer Engineering, The University of Suwon, Hwasungshi, Korea \\ ${ }^{2}$ Intelligent Textile System Research Center, Seoul, Korea \\ ${ }^{3}$ Department of Chemistry, The University of Suwon, Hwasungshi, Korea \\ ${ }^{4}$ Materials Science and Engineering, Macromolecular Science and Engineering Center, The University of Michigan, \\ Ann Arbor, Michigan 48109 \\ ${ }^{5}$ Venture and Core RED, Analytical Sciences Laboratory, Dow Chemical, Midland, Michigan 48667
}

Received 25 February 2007; accepted 3 May 2007

DOI 10.1002/app.26721

Published online 27 June 2007 in Wiley InterScience (www.interscience.wiley.com).

\begin{abstract}
Nanocomposites from polyurethane (PU) and montmorillonite (MMT) were prepared under meltmixing condition, by a twin screw extruder along with a compatibilizer to enhance dispersion of MMT. MMT used in this study was Cloisite 25A (modified with dimethyl hydrogenated tallow 2-ethylhexyl ammonium) or Cloisite 30B (modified with methyl tallow bis-2-hydroxyethyl ammonium). Maleic anhydride grafted polypropylene (MAPP) was used as the compatibilizer. XRD and TEM analysis demonstrated that melt mixing by a twin-screw extruder was effective in dispersing MMT through the PU matrix. The PU/Cloisite 30B composite exhibited better
\end{abstract}

interlayer separation than the PU/Cloiste 25A composite. Nanoparticle dispersion was the best at $1 \mathrm{wt} \%$ of MMT and improved with compatibilizer content for both composites. Properties of the composites such as complex viscosity and storage modulus were higher than that of a pure PU matrix and increased with the increase in MMT content, but decreased with the increase in compatibilizer content. (c) 2007 Wiley Periodicals, Inc. J Appl Polym Sci 106: 712721, 2007

Key words: nanocomposite; twin-screw extruder; melt mixing; montmorillonite; compatibilizer

\section{INTRODUCTION}

Nanocomposites of polymer and layered silicate are known to demonstrate superior reinforcement with minimum sacrifice of strain and impact strength when compared with normal composites employing a small amount of reinforcing material. In addition, other desirable characteristics such as low gas permeability, good thermal stability, and flame retardation are observed. ${ }^{1}$ Light weight and economic competitiveness using a minimal amount of reinforcing material are other advantages of nanocomposites. Nanometer scale particles used in polymer nanocomposites include silicate (phyllosilicate or layered silicate), POSS (nanostructured polyhedral oligomeric silsesquioxanes), carbon nanotubes, and metal or inorganic nanoparticles. Layered silicate is a commonly used as nanoparticle. Layered silicate, composed of silicon, alumina, and magnesium oxide, is classified as montmorillonite (MMT), saponite, hec-

Correspondence to: B. C. Chun (bcchun@suwon.ac.kr).

Contract grant sponsor: Korea Science and Engineering Foundation (KOSEF); contract grant number: R11-2005-065.

Journal of Applied Polymer Science, Vol. 106, 712-721 (2007) C 2007 Wiley Periodicals, Inc.

\section{(\$ILEY}

InterScience torite, vermiculite, mica, talc, and kaolinite depending on the composition, structure, and layer ratio. MMT is favored among the layered silicates for polymer nanocomposites. Because MMT is hydrophilic and less compatible with polymer matrices, $\mathrm{Na}^{+}$in MMT is substituted with hydrophobic surfactant before processing to improve compatibility and better layer separation. ${ }^{2}$ Two types of morphology, intercalation and exfoliation, are proposed for the MMT nanocomposite. Intercalation is a highly organized multilayered structure where the polymer chain is interspersed between each MMT layer, thus resulting in more separated layers. Exfoliation is the further intersection of a polymer chain deep into the interspace of MMT layers, terminating ionic interaction between each MMT layer. $d$-Spacing (basal spacing) between MMT layers can be determined from XRD analysis and differentiates the intercalation from the exfoliation. Peaks corresponding to interlayer distance between MMT layers, in the case of intercalation, appear in the low angle range, whereas such peaks disappear in exfoliated structure due to the large interlayer distance $(10 \mathrm{~nm}){ }^{3}$

As a strategy for nanocomposite preparation, one can penetrate monomers into the silicate layers and then polymerize ${ }^{4,5}$ or directly insert polymer chains 
TABLE I

Composition of PU/MMT Nanocomposite

\begin{tabular}{lccc}
\hline \multicolumn{1}{c}{ Sample code } & $\begin{array}{c}\text { Polyurethane } \\
\text { (wt \%) }\end{array}$ & $\begin{array}{c}\text { Montmorillonite }^{\mathrm{a}} \\
(\mathrm{MMT}, \text { wt } \%)\end{array}$ & $\begin{array}{c}\text { Compatibilizer }^{\mathrm{b}} \\
\text { (MAPP, wt \%) }\end{array}$ \\
\hline PU matrix & 100 & 0 & 0 \\
PU99/MMT1 & 99 & 1 & 0 \\
PU97/MMT3 & 97 & 3 & 0 \\
PU95/MMT5 & 95 & 5 & 0 \\
PU99/MMT1/MAPP2 & 99 & 1 & 2 \\
PU97/MMT3/MAPP2 & 97 & 3 & 2 \\
PU95/MMT5/MAPP2 & 95 & 5 & 4 \\
PU99/MMT1/MAPP4 & 99 & 1 & 4 \\
PU97/MMT3/MAPP4 & 97 & 3 & 4 \\
PU95/MMT5/MAPP4 & 95 & 5 & 4 \\
\hline
\end{tabular}

a Cloisite 25A or Cloisite 30B.

${ }^{\mathrm{b}}$ Maleic anhydride polypropylene (PP-g-MAH).

into the silicate layers. ${ }^{6}$ The direct polymer insertion method can be either solution or melt-mixing method. ${ }^{7,8}$ Melt mixing is more difficult than other methods when inserting highly viscous polymer chains into the MMT interlayers. However, the simplicity of melt-mixing method is advantageous over other methods for practical reasons. In this article, we investigate PU/MMT nanocomposite prepared by the melt-mixing method together with compatibilizer and focus on MMT dispersion, mechanical, and rheological properties with a goal of finding a nanocomposite with enhanced mechanical properties.

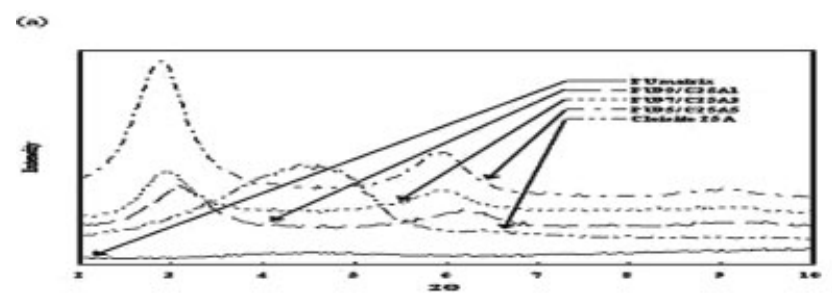

क)

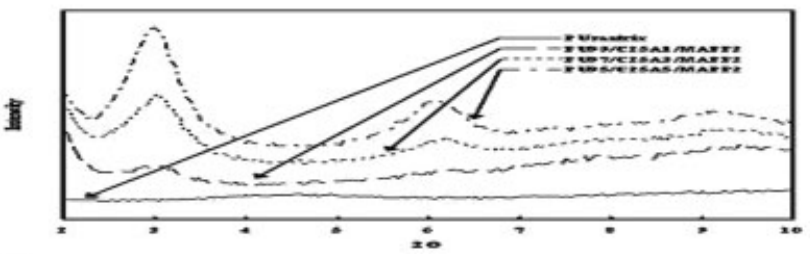

$\Leftrightarrow$

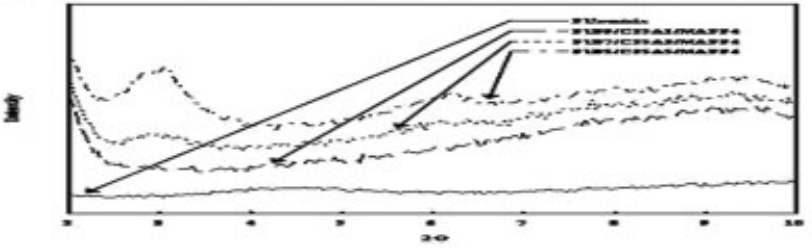

Figure 1 XRD spectra of PU/MMT (cloisite 25A) nanocomposite with various MAPP content: (a) 0\% MAPP, (b) $2 \%$ MAPP, and (c) $4 \%$ MAPP.

\section{EXPERIMENTAL}

\section{Materials}

Polyurethane (PU) was purchased from Kolon Petrochemical $\left(M_{w}=100,000 \mathrm{~g} / \mathrm{mol}\right.$, grade: Ellas K787A), and compatibilizer was from Honam Petrochemical (PP-g-MAH, graft ratio $=1 \%, M_{w}$ $=40,000-50,000 \mathrm{~g} / \mathrm{mol}$, grade: CM-1120). Layered silicate MMT from Nanoclay were Cloisite 25A (MMT modified with dimethyl hydrogenatedtallow 2-ethylhexyl ammonium) and Cloisite 30B (MMT modified with methyl tallow bis-2-hydroxyethyl ammonium),



()

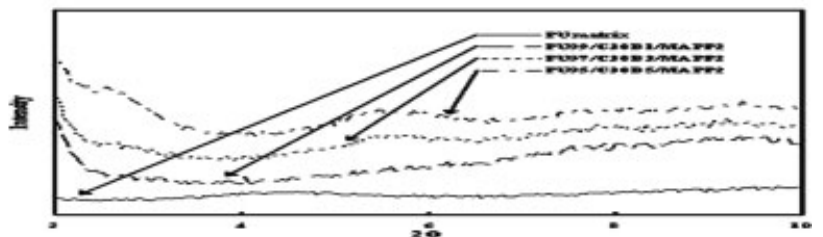

$\Leftrightarrow$

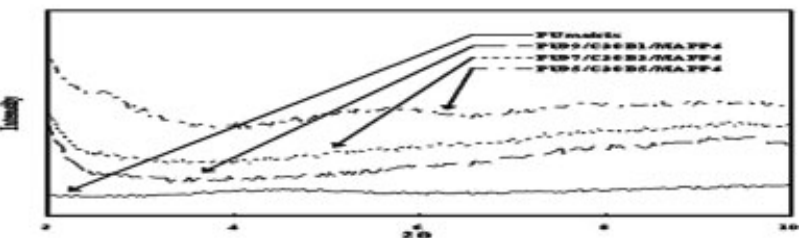

Figure 2 XRD spectra of PU/MMT (Cloisite 30B) nanocomposite with various MAPP content: (a) $0 \%$ MAPP; (b) $2 \%$ MAPP; and (c) $4 \%$ MAPP. 

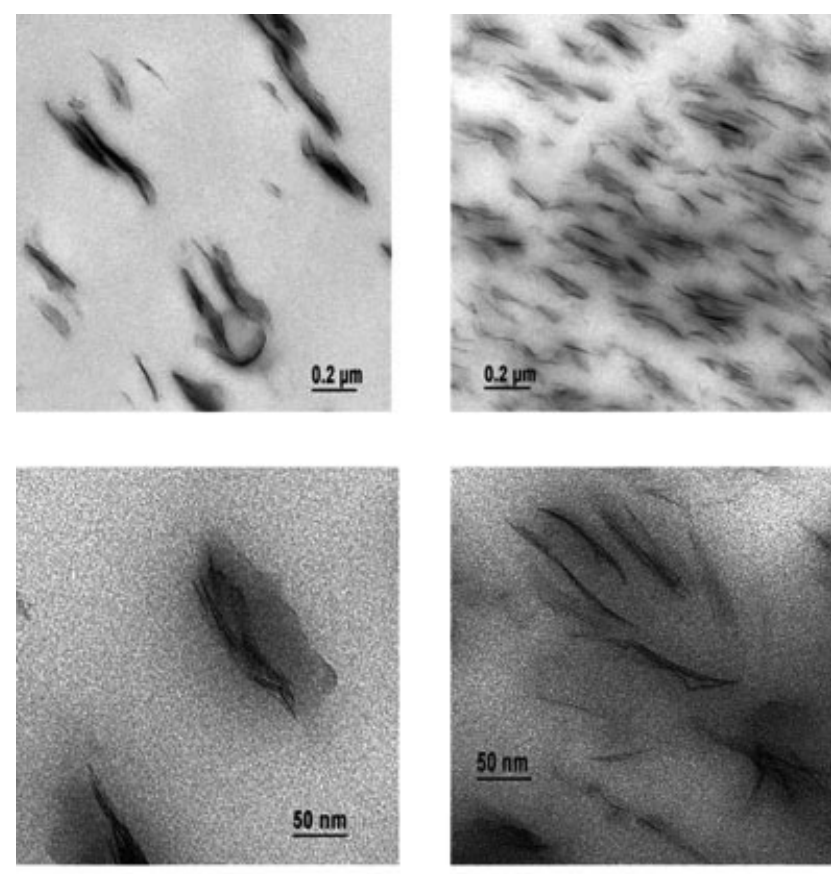

(a)

(b)

Figure 3 TEM micrographs of PU/MMT nanocomposite: (a) PU/C25A; (b) PU/C30B. and their ion exchange capacities (CEC) were 95 meqiv/100 g and 90 meqiv/100 g, respectively.

\section{Preparation of nanocomposite}

PU/MMT nanocomposite was prepared by co-rotating twin screw extruder (Bau Tech, BA-11, screw diameter $=11 \mathrm{~mm}, L / D=40)$ with extruder temperature profile between 210 and $225^{\circ} \mathrm{C}$, screw torque of $2.5 \mathrm{Nm}$, and screw speed of $300 \mathrm{rpm}$. Detailed composition of the nanocomposite is listed in Table I.

\section{Measurements}

MMT dispersion within PU/MMT matrix was analyzed by wide angle X-ray diffractometry (WAXD, Rint 2000, Rigaku Denki) of a 1-mm thick sheet under operating conditions of $40 \mathrm{kV}, 30 \mathrm{~mA}, 1^{\circ} / \mathrm{min}$ scanning speed with $2 \theta=2^{\circ}-15^{\circ}$, and a Ni-filtered $\mathrm{Cu}$ $\mathrm{K} \alpha \mathrm{X}$-ray source. Samples for transmission electron microscopy (TEM) were cut from a microtensile specimen perpendicular to the injection mould direction. The specimen block was thin-sectioned at $-110^{\circ} \mathrm{C}$ by Leica UC6 ultracryomicrotome. A thin section $\sim 95 \mathrm{~nm}$ in thickness was collected onto a copper grid and
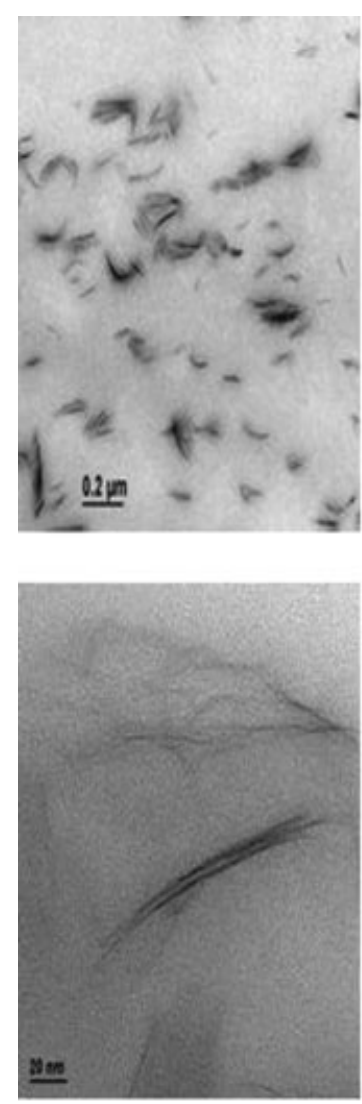

(a)
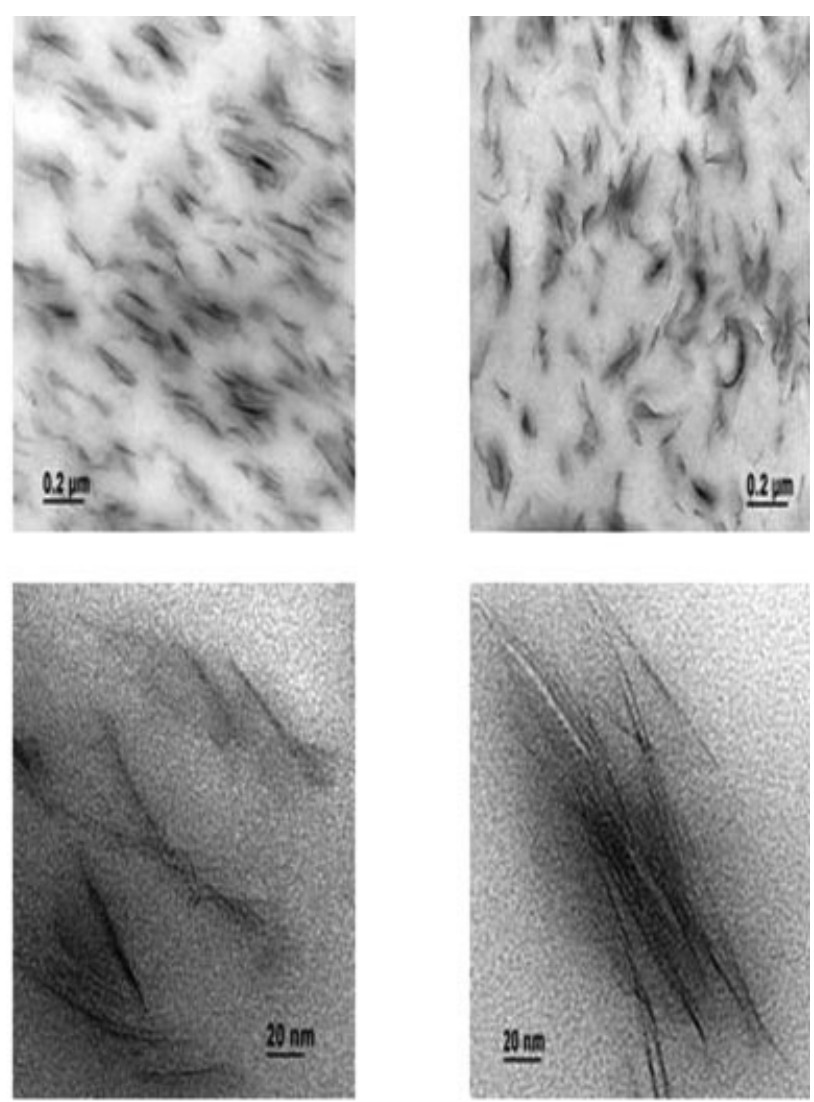

(b)

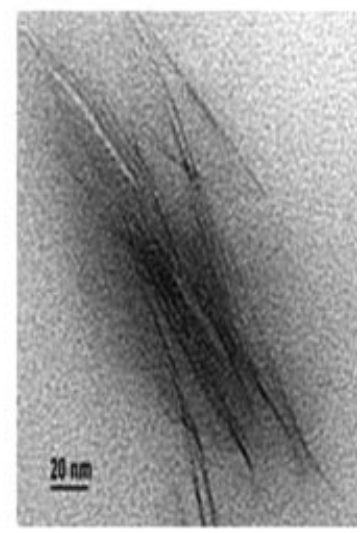

(c)

Figure 4 TEM micrographs of PU/MMT nanocomposite with various MMT (Cloisite 30B) content: (a) PU99/MMT1; (b) PU97/MMT3; (c) PU95/MMT5. 


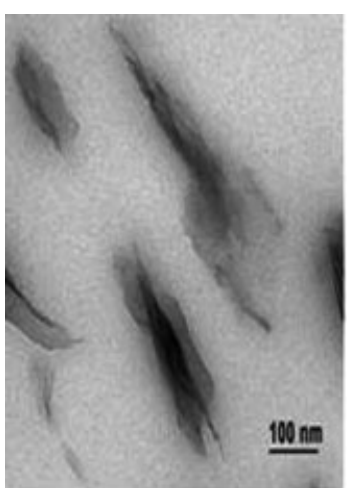

(a)



(b)

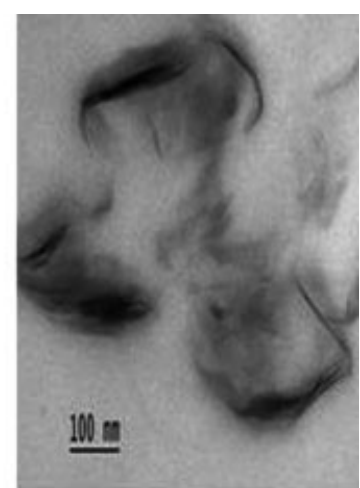

(c)



(d)



(e)

Figure 5 TEM micrographs of PU/MMT (Cloisite 25A) nanocomposite: (a) PU97/C25A3; (b) PU99/C25A1/MAPP2; (c) PU97/C25A3/MAPP2; (d) PU95/C25A5/MAPP2; (e) PU97/C25A3/MAPP4.

examined with JEOL JEM1230 TEM at 120-kV accelerating voltage. The image was recorded digitally with a Gatan Multiscan CCD camera (Model 749). To measure the degree of hydrogen bonding in the nanocomposite, FTIR (FTIR 4200, Jasco) spectra were scanned with 50 accumulations and $4 \mathrm{~cm}^{-1}$ resolution. The tensile strength was measured according to ASTM D 638 using a universal testing machine (LR $50 \mathrm{~K}$, Lloyd Instrument, UK) with a gauge length of $25 \mathrm{~mm}$ and a crosshead speed of $10 \mathrm{~mm} / \mathrm{min}$. Rheological properties (complex viscosity $\left(\eta^{*}\right)$ and storage modulus $\left(G^{\prime}\right)$ ) were measured by a modular compact rheometer (Anton Paar, MCR300) at $180^{\circ} \mathrm{C}, 10 \%$ of strain, and $0.01-1001 / \mathrm{s}$ of angular frequency, and the specimen ( $25 \mathrm{~mm}$ diameter and $2 \mathrm{~mm}$ thickness) was prepared by a thermal press.

\section{RESULTS AND DISCUSSION}

X-ray diffraction and TEM can determine how well a polymer chain penetrated the interlayer of silicate and TEM especially visualizes in nanometer scale the dispersion of the layered silicate in the polymer nanocomposite.

XRD spectra of PU/MMT nanocomposites showed the characteristic MMT peak at $2 \Theta=4.76^{\circ}$ for both types of MMT (Cloisite 25A or 30B) and a peak shift to lower angles after melt mixing. A peak shift from $2 \Theta=4.76^{\circ}(18.6 \AA)$ to $2 \Theta=2.88^{\circ}-3.08^{\circ}$ (28.7-30.7 $\AA$ ) correlates to an increase of the interlayer distance of PU/C25A nanocomposite by more than $10.1 \AA$ (Fig. 1). Because the characteristic MMT peak shifted from $2 \Theta=4.76^{\circ}(18.6 \AA)$ to $2 \Theta=2.44^{\circ}$ (36.2 $\AA$ ), the interlayer distance of PU/C30B nanocomposite increased about $17.7 \AA$ (Fig. 2). The significant increase in $d$-spacing suggested that PU polymer chain intercalated into the MMT layer. ${ }^{9-11}$ The XRD peak intensity increases with MMT content and the absence of any peak for PU supports the fact that the diffraction peaks originate from MMT. Cloisite 30B was more susceptible to the intercalation of the polymer chain than Cloisite 25A, considering the $75 \%$ larger $d$-spacing under similar 


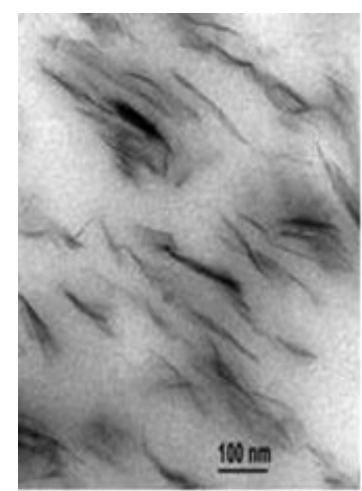

(a)

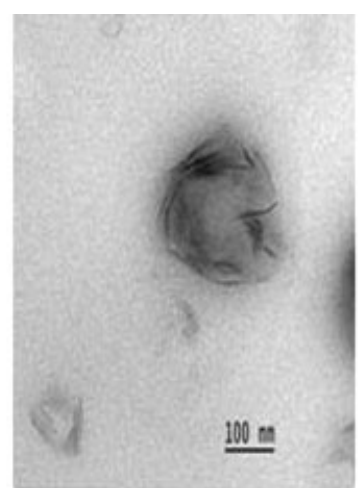

(b)

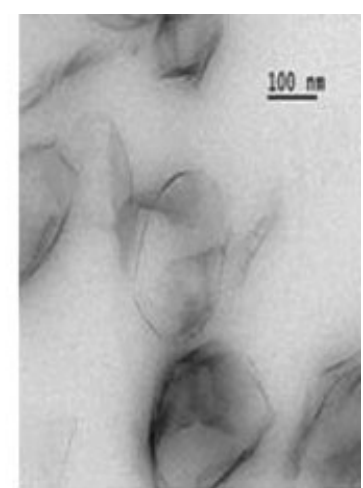

(c)

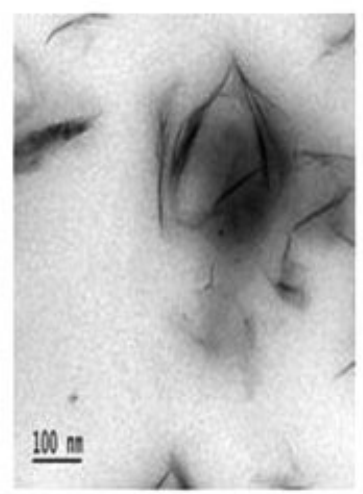

(d)

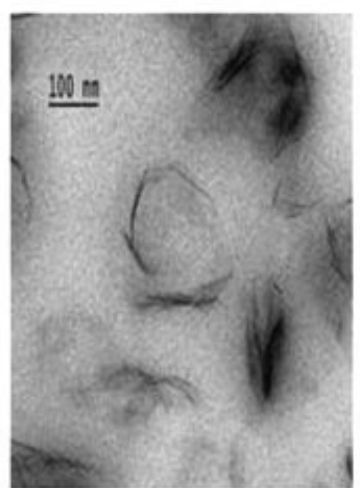

(e)

Figure 6 TEM micrographs of PU/MMT (Cloisite 30B) nanocomposite: (a) PU97/C30B3; (b) PU99/C30B1/MAPP2; (c) PU97/C30B3/MAPP2; (d) PU95/C30B5/MAPP2; (e) PU97/C30B3/MAPP4.

conditions. The measured peak intensity of Cloisite 30B was lower than Cloisite 25A with equal MMT content implying better compatibility of Cloisite 30B with PU. Because the modifying reagent of Cloisite 30B includes hydroxyl groups it is more hydorophilic than Cloisite 25A and the hydrophilic surface of Cloisite 30B eases the PU chain penetration into the MMT interlayer. Control of surface polarity through careful selection of the modifying reagent plays an important role in the dispersion of MMT and the resultant mechanical properties of the nanocomposite. $^{12}$ Another peak around $2 \Theta=5^{\circ}$ originates from the decomposition of reagent under hot melt-mixing condition yielding MMT devoid of modifying reagent, which reduces the interlayer spacing. Melt mixing via internal mixer normally takes more than $10 \mathrm{~min}$ of processing, but the extruder method used for our experiment significantly shortens the processing time and reduces the risk of decomposition of heat-labile compound. During our extrusion processing, residence time in screw was about $100 \mathrm{~s}$ at $300 \mathrm{rpm}$, and could be shortened to
$65 \mathrm{~s}(400 \mathrm{rpm})$ or $47 \mathrm{~s}(500 \mathrm{rpm})$ to reduce the risk of decomposition.

Diffraction peaks become blurry with the inclusion of compatibilizer for both MMTs, and the peaks almost disappear at 4 wt \% of MAPP (compatibilizer) for all of the nanocomposites. Compatibilizer is added to improve MMT dispersion, which affects Cloisite 30B more than Cloisite 25A. MAPP has a maleic anhydride group attached to polypropylene (PP-g-MAH) that is compatible with the hydroxyl group. MAPP is compatible with Cloisite 30B because, unlike Cloisite 25A, Cloisite 30B has a hydroxyl group in the modifying reagent. Therefore, Cloisite 30B disperses better with the compatibilizer in nanocomposite and XRD peaks become more blurry at the higher compatibilizer content. ${ }^{13}$

TEM of the nanocomposite was taken to confirm our understanding of MMT dispersion based on XRD results. Cloisite 30B was also found to disperse better than Cloisite 25A in nanocomposites, as expected from XRD results. In magnified figures, the layer of Cloisite 30B disperses well in the PU matrix, 


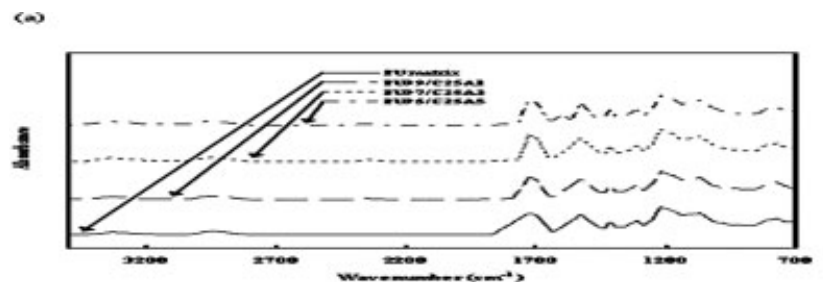

())

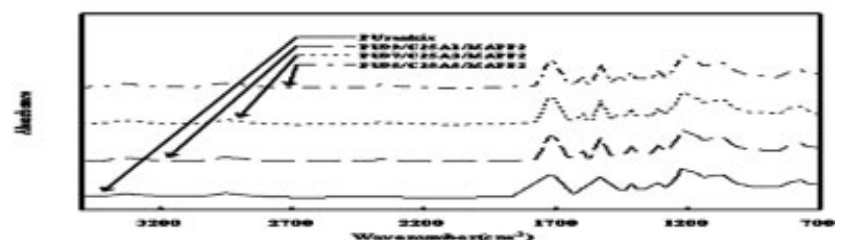

$\Leftrightarrow$

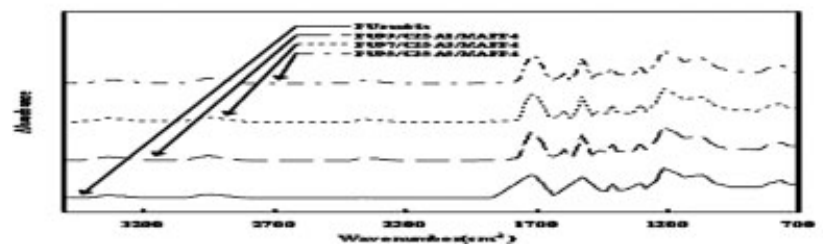

Figure 7 Infrared spectra of PU/MMT (Cloisite 25A) nanocomposite with various MAPP content: (a) 0 wt \% MAPP; (b) 2 wt \% MAPP; (c) 4 wt \% MAPP.

but Cloisite 25A layer aggregates. The two contrasting results demonstrate that Cloistie $30 \mathrm{~B}$ is more compatible with PU than Cloisite 25A due to

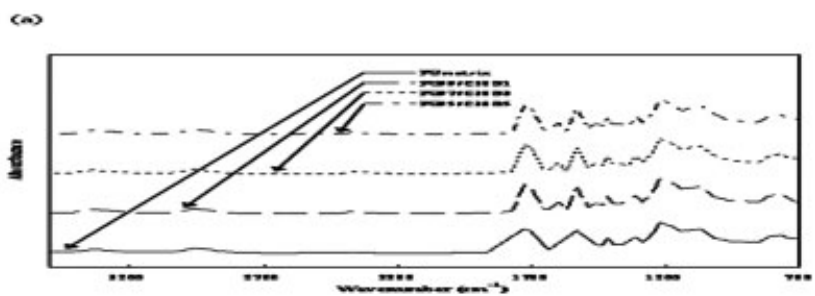

())

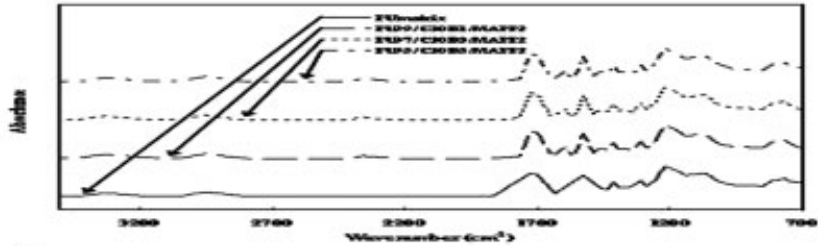

$\Leftrightarrow$

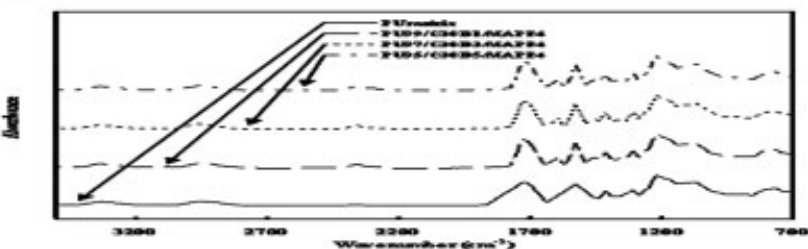

Figure 8 Infrared spectra of PU/MMT (Cloisite 30B) nanocomposite with various MAPP content: (a) 0 wt \% MAPP; (b) 2 wt \% MAPP; (c) 4 wt \% MAPP.
TABLE II

Carbonyl Hydrogen Bonding Index (R), Degree of Phase Separation, and Degree of Phase Mixing of PU/MMT (Cloisite 25A) Nanocomposite

\begin{tabular}{lccc}
\hline \multicolumn{1}{c}{ Sample code } & $R\left(A_{1703} / A_{1733}\right)^{\mathrm{a}}$ & DPS & DPM \\
\hline PU Matrix & 0.936 & 0.483 & 0.517 \\
PU99/C25A1 & 0.950 & 0.487 & 0.513 \\
PU97/C25A3 & 0.939 & 0.484 & 0.516 \\
PU95/C25A5 & 0.939 & 0.484 & 0.516 \\
PU99/C25A1/MAPP2 & 0.946 & 0.486 & 0.514 \\
PU97/C25A3/MAPP2 & 0.935 & 0.483 & 0.517 \\
PU95/C25A5/MAPP2 & 0.934 & 0.483 & 0.517 \\
PU99/C25A1/MAPP4 & 0.937 & 0.484 & 0.516 \\
PU97/C25A3/MAPP4 & 0.937 & 0.484 & 0.516 \\
PU95/C25A5/MAPP4 & 0.931 & 0.482 & 0.518
\end{tabular}

DPS, degree of phase separation; DPM, degree of phase mixing.

${ }^{a} \mathrm{~A}_{1703}$, absorption of hydrogen-bonded carbonyl; $\mathrm{A}_{1733}$, absorption of free carbonyl.

hydroxyl group of modifying reagent on Cloisite 30B (Fig. 3). As MMT (Cloisite 30B) content increases, MMT disperses well in PU matrix, although some aggregation appears at $5 \mathrm{wt} \%$ of MMT (Fig. 4). From TEM results, compatibilizer also improved the MMT dispersion for both Cloisite 25A and Cloisite 30B. MMT (Cloisite 25A) dispersion at the same Cloisite 25A content gradually improves with compatibilizer content [compare Fig. 5(a,c,e)], and Cloisite 30B [compare Fig. 6(a,c,e)] follows a similar trend. TEM reveals that Cloisite 30B disperses better than Cloisite 25A and compatiblizer helps MMT dispersion in PU matrix. The degree of phase separation (DPS) between PU and MMT was compared from FTIR spectra for the whole range of nanocomposites (Figs. 7 and 8). Strong interaction between PU and MMT layer may affect MMT dispersion in

TABLE III

Carbonyl Hydrogen Bonding Index $(R)$, Degree of Phase Separation, and Degree of Phase Mixing of PU/MMT (Cloisite 30B) Nanocomposite

\begin{tabular}{lccc}
\hline \multicolumn{1}{c}{ Sample code } & $R\left(A_{1703} / A_{1733}\right)^{\mathrm{a}}$ & DPS & DPM \\
\hline PU Matrix & 0.936 & 0.483 & 0.517 \\
PU99/C30B1 & 0.942 & 0.485 & 0.515 \\
PU97/C30B3 & 0.940 & 0.485 & 0.515 \\
PU95/C30B5 & 0.950 & 0.487 & 0.513 \\
PU99/C30B1/MAPP2 & 0.944 & 0.486 & 0.514 \\
PU97/C30B3/MAPP2 & 0.947 & 0.485 & 0.514 \\
PU95/C30B5/MAPP2 & 0.942 & 0.485 & 0.515 \\
PU99/C30B1/MAPP4 & 0.938 & 0.484 & 0.516 \\
PU97/C30B3/MAPP4 & 0.941 & 0.485 & 0.515 \\
PU95/C30B5/MAPP4 & 0.945 & 0.486 & 0.514
\end{tabular}

DPS, degree of phase separation; DPM, degree of phase mixing.

a $\mathrm{A}_{1703}$, absorption of hydrogen-bonded carbonyl; $\mathrm{A}_{1733}$, absorption of free carbonyl. 
(a)

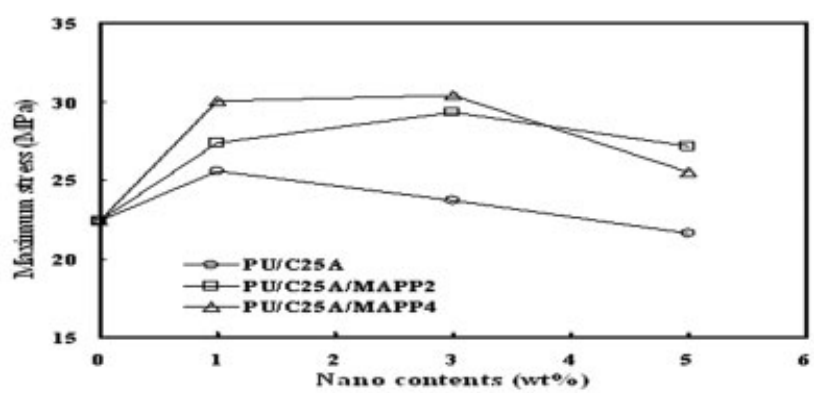

(b)

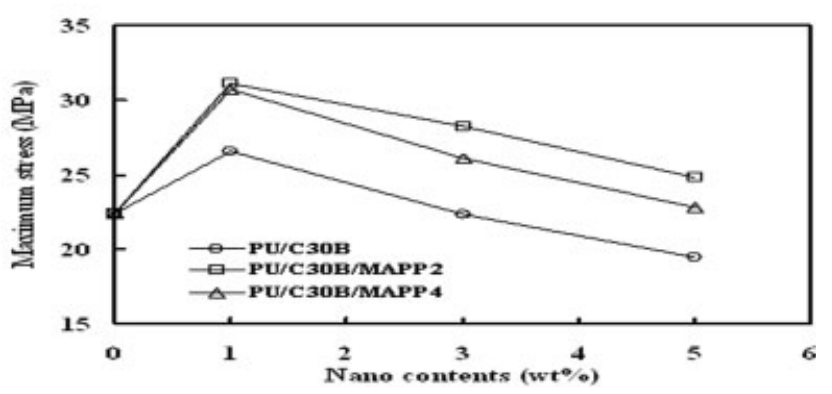

Figure 9 Maximum stress of $\mathrm{PU} / \mathrm{MMT}$ nanocomposite with various MMT content: (a) PU/C25A; (b) PU/C30B.

PU matrix and induce the phase separation of PU. ${ }^{14}$ The fact that the IR band corresponding to the free carbonyl group appears at $1733 \mathrm{~cm}^{-1}$ and the hydrogen-bonded carbonyl group shifts to $1703 \mathrm{~cm}^{-1}$ can be used to determine the fraction of hydrogen-

(a)



(b)

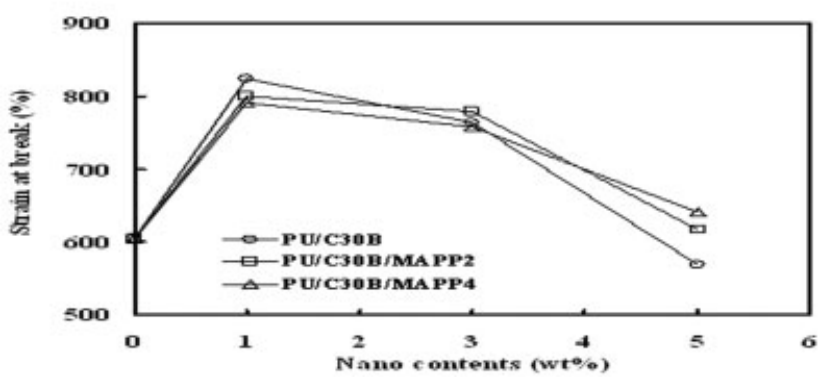

Figure 10 Strain at break of PU/MMT nanocomposite with various MMT content: (a) PU/C25A; (b) PU/C30B. bonded phase of PU from IR spectra: hydrogen bonding index $(R)$ in eq. (1) is compared for each $\mathrm{PU} / \mathrm{MMT}$ nanocomposite.

$$
R=A_{1703} / A_{1733}=C_{\text {bonded }} \varepsilon_{\text {bonded }} / C_{\text {free }} \varepsilon_{\text {free }}
$$

where $A$ is absorbance and $C$ is concentration; $\varepsilon_{\text {bonded }}$ and $\varepsilon_{\text {free }}$ are the extinction coefficients of bonded and free carbonyl group, and $\varepsilon_{\text {bonded }} / \varepsilon_{\text {free }}$ is generally 1.0-1.2. ${ }^{15,16}$ The DPS (degree of hydrogen bonded phase of PU) and degree of phase mixing (DPM, degree of unbound phase of PU) are calculated from $R$ by eqs. (2) and (3), ${ }^{15-17}$ and are summarized in Tables II and III.

$$
\begin{gathered}
\text { DPS }=C_{\text {bonded }} /\left(C_{\text {bonded }}+C_{\text {free }}\right)=R /(R+1) \\
\text { DPM }=1-\text { DPS }
\end{gathered}
$$

No apparent difference in DPS and DPM between PU/MMT and PU matrix is observed and hydrogen bonding, therefore, is not affected by the presence of MMT. Such result is quite different from the one reported by $\mathrm{Wei}^{14}$ who prepared PU/MMT nanocomposite by solution mixing. The melt-mixing method adopted in this investigation and the type of PU may be responsible for the difference. The method, solution, or melt mixing, selected for nanocomposite preparation depends on the requirements for final application and the comparison of both methods for a range of nanocomposites is needed.

(a)

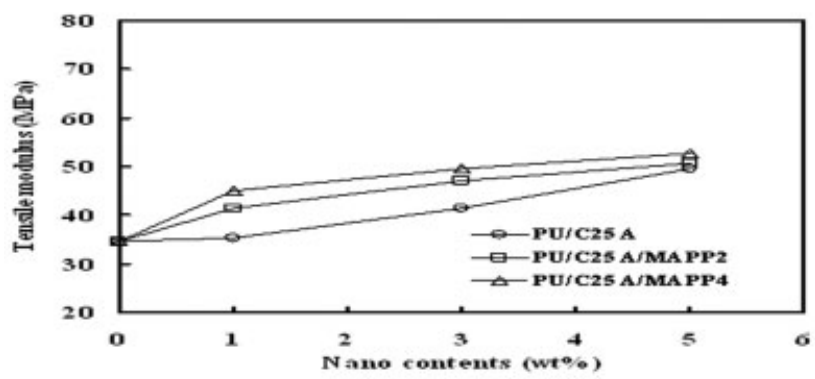

(b)

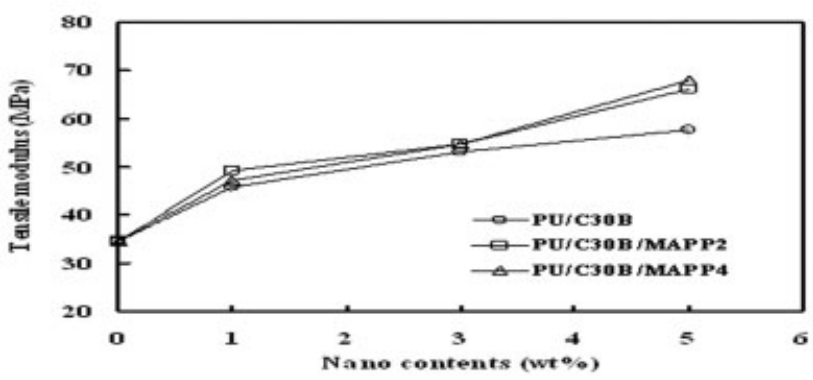

Figure 11 Tensile modulus of PU/MMT nanocomposite with various MMT content: (a) PU/C25A; (b) PU/C30B. 
(a)
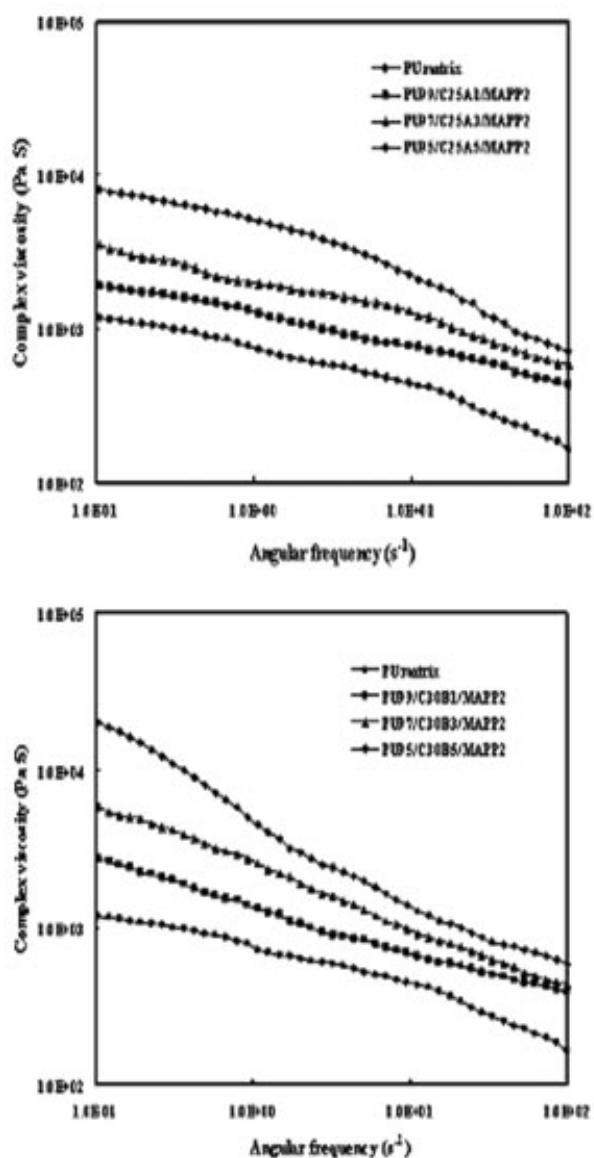

(b)


Figure 12 Complex viscosity versus angular frequency for PU/C25A and PU/C30B nanocomposite with various (a) MMT content, and (b) MAPP content.

Maximum stress is the highest at 1 wt \% MMT, and decreases with the further increase of MMT content (Fig. 9). Gradual aggregation of the MMT layers, already shown by TEM, at high MMT content reduces the tensile strength. Compatibilizer increases tensile strength for the entire range of MMT (both Cloisite 25A and Cloisite 30B). Strain at break is at a maximum value at $1 \mathrm{wt} \% \mathrm{MMT}$ and decreases at the high MMT contents. Compatibilizer improves only the strain of Cloisite 25A nanocomposite. Cloisite 30B nanocomposite exhibits similar strain with and without compatibilizer (Fig. 10). Because the strain of Cloisite 30B nanocomposite without compatibilizer is already higher than that of Cloisite 25A nanocomposite, the addition of compatibilizer to Cloisite 30B nanocomposite does not exhibit a significant increase like Cloisite 25A. Modifying agent on MMT surface reduces the interfacial segregation of PU and MMT and lubricates PU extension against MMT layer during the tensile test. ${ }^{18}$ Modulus of elasticity increases in proportion to MMT content. Modulus of Cloisite 25A nanocomposite improves with the addition of compatibilizer, while Cloisite 30B nanocomposite is not affected by compatibilizer (Fig. 11). ${ }^{19}$ Similarity in both modulus and strain data is due to the presence (Cloisite 30B) or the absence (Cloisite 25A) of a hydroxyl group on MMT that makes hydrogen bonding with PU chains. Rheological property is helpful in understanding the morphology of the nanocomposite in the melted state. Morphological change and residual stress distribution during melt-processing affect other physical properties. Rheological properties of the polymer/ MMT nanocomposites can be significantly influenced by shear stress during melt-mixing process and the resultant MMT dispersion. ${ }^{20}$ Complex viscosity of the PU matrix and the PU/MMT nanocomposite under varying frequency is investigated, while MMT and compatibilizer content is changed (Fig. 12). Complex viscosity of PU/MMT nanocomposite is higher than PU matrix because the intercalated or exfoliated nanocomposite drags polymer movement. $^{20,21}$ During the complex viscosity experiment, shear thinning is observed as the shear 
(a)


(b)
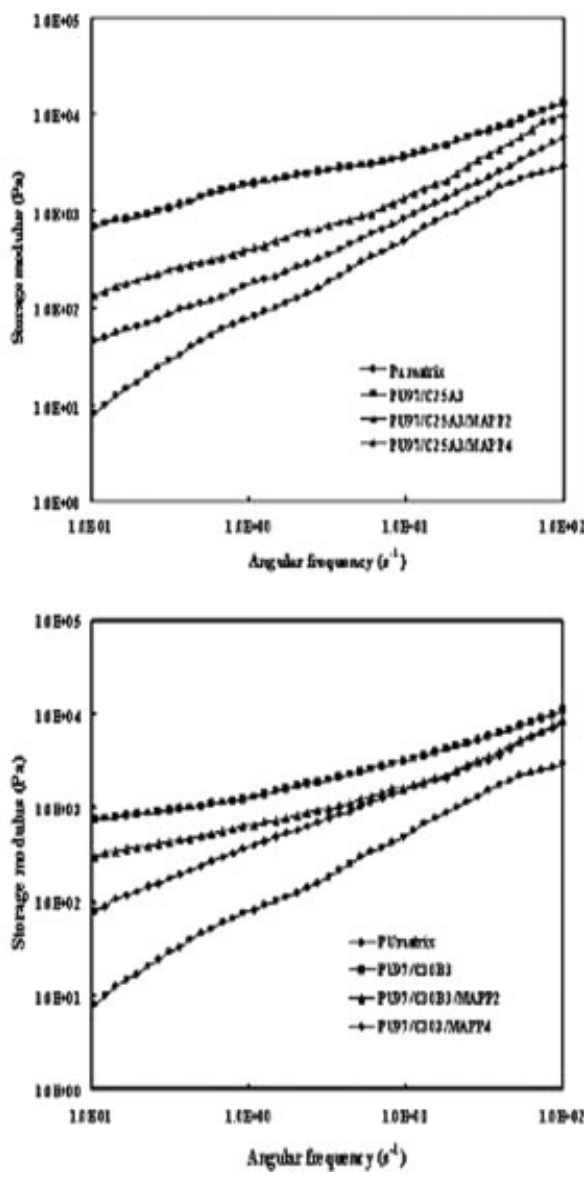

Figure 13 Storage modulus vs. angular frequency for PU/C25A and PU/C30B nanocomposite with various (a) MMT content, and (b) MAPP content.

stress increases. ${ }^{22}$ The increased complex viscosity of $\mathrm{PU} / \mathrm{MMT}$ nanocomposite is reduced by the addition of compatibilizer. The storage modulus of a nanocomposite with different MMT content [Fig. 13(a)] and compatibilizer [Fig. 13(b)] is investigated. Addition of MMT increases the storage modulus for the same reason as the increase in complex viscosity. ${ }^{20}$ The storage modulus of the nanocomposite moderately increased with MMT content, but decreased as the compatibilizer content increased for both types of MMT. Slope of the storage modulus over frequency decreases for the nanocomposite compared to PU matrix. The similar increase of the complex viscosity and storage modulus with MMT content and decrease with compatibilizer content occur because MMT attracts and reinforces the polymer chain as a nucleus and the compatibilizer reduces attraction by interfering with the contact between MMT and PU. Overall, the tensile strength and strain of PU has improved with the addition of MMT and compatibilizer, but there is still a room for quantum leap of mechanical properties of nanocomposite.

\section{CONCLUSION}

PU/MMT nanocomposite is prepared with MMT and PU by melt mixing together with compatibilizer to enhance MMT dispersion. Cloisite 30B yields better dispersion and less aggregation than Cloisite 25A in a PU matrix. The nanocomposite with $1 \mathrm{wt} \%$ of MMT exhibited the best mechanical properties for both types of MMT. The nanocomposite with Cloisite $25 \mathrm{~A}$ is significantly affected by the compatibilizer in contrast to Cloisite 30B, which demonstrated almost no dependence on the compatibilizer content. The complex viscosity and storage modulus of the nanocomposite increased with MMT content and decreased with comapatibilizer content. The meltmixing method for the preparation of the nanocomposite is convenient, requires short processing time, is low cost, poses little environmental hazard from volatile organic solvents, and yields good dispersion of MMT. The melt-mixing method may be advantageous over other mixing methods using an internal mixer or a two-roll mill considering the risk of com- 
posite degradation during the process. Enhanced mechanical properties, good dispersion of MMT, and the control of complex viscosity and storage modulus by the addition of compatibilizer are the positive results from our nanocomposite research.

The authors of this paper would like to thank the Korea Science and Engineering Foundation (KOSEF) for supporting this research through the SRCIERC program of MOST/KOSEF (R11-2005-065).

\section{References}

1. Giannelis, E. P. Adv Mater 1996, 8, 29.

2. Lee, Y. H.; Hong, S. K.; Yoon, K. S.; Choi, I. S.; Lee, S. G.; Lee, J. H.; Choi, K. Y. Polymer (Korea) 2001, 25, 818.

3. Gilman, J. W. Appl Clay Sci 1999, 15, 31.

4. Lee, D. C.; Jang, L. W. J Appl Polym Sci 1996, 61, 1117.

5. Messersmith, P. B.; Giannelis, E. P. Chem Mater 1993, 5, 1064.

6. Choi, H. K.; Park, Y. H.; Lyu, S. G.; Kim, B. S.; Sur, G. S. Polymer (Korea) 1999, 23, 456.

7. Vaia, R. A.; Jandt, K. D.; Kramer, E. J.; Giannelis, E. P. Macromolecules 1995, 28, 8080 .
8. Vaia, R. A.; Giannelis, E. P. Macromolecules 1997, 30, 7990.

9. Hsiao, S. H.; Liou, G. S.; Chang, L. M. J Appl Polym Sci 2001, 80, 2067.

10. Zilg, C.; Mulhaupt, R.; Finter, J. Macromol Chem Phys 1999, 200, 661 .

11. Ke, Y.; Lu, J.; Yi, X.; Zhao, J.; Qi, Z. J Appl Polym Sci 2000, 78, 808.

12. Chang, W. Y.; Lee, K. J.; Nam, J. D. Polymer (Korea) 2003, 27, 75.

13. Kawasumi, M.; Hasegawa, N.; Kato, M.; Usuki, A.; Okada, A. Macromolecules 1997, 30, 6333.

14. Tien, Y. I.; Wei, K. H. Polymer 2001, 42, 3213.

15. Seymour, R. W.; Estes, G. M.; Cooper, S. L. Macromolecules 1970, 3, 579.

16. Chang, J. H.; Park, D. K.; Ihn, K. J. J Polym Sci Part B: Polym Phys 2001, 39, 471.

17. An, Y. U.; Chang, J. H.; Park, Y. H.; Park, J. M. Polymer (Korea) 2002, 26, 381.

18. Lee, S. U.; Oh, I. H.; Lee, J. H.; Choi, K. Y.; Lee, S. G. Polymer (Korea) 2005, 29, 271.

19. Wang, Z.; Pinnavaia, T. J. Chem Mater 1998, 10, 3769.

20. Solomon, M. J.; Almusallam, A. S.; Seefelt, K. F.; Somwangthanaroj, A.; Varadan, P. Macromolecules 2001, 34, 1864.

21. Krishnamoorti, R.; Vaia, R. A.; Giannelis, E. P. Chem Mater 1996, 8, 1728.

22. Krishnamoorti, R.; Giannelis, E. P. Macromolecules 1997, 30, 4097.

23. Lim, Y. T.; Park, O. O. Rheol Acta 2001, 40, 220. 